\title{
KRAB-containing zinc finger gene ZNF268 encodes multiple alternatively spliced isoforms that contain transcription regulatory domains
}

\author{
HUANJIE SHAO, CHENGANG ZHU, ZHOUZHOU ZHAO, MINGXIONG GUO, \\ HONGLING QIU, HUI LIU, DI WANG, LU XUE, LI GAO, CHONG SUN and WENXIN LI
}

State Key Laboratory of Virology, College of Life Sciences, Wuhan University, Wuhan 430072, P.R. China

Received March 7, 2006; Accepted April 19, 2006

\begin{abstract}
The ZNF268 gene was originally isolated from an early human embryo cDNA library. Several different transcripts have been isolated for the ZNF268 gene and developmental expression studies suggest that ZNF268 plays a role in the development of human fetal liver and the differentiation of blood cells. In our effort to study the functions of ZNF268 in different organs during development and in pathogenesis, we have now identified 3 novel splicing isoforms, ZNF268e, ZNF268f and ZNF268g, in human fetal tissues and human tumor derived cell lines. The 8 alternatively spliced mRNAs discovered so for are predicted to encode 3 protein isoforms. Expression analysis showed that different mRNA isoforms have different expression profiles. In particular, ZNF268c mRNA was detected only in tumor cells, and ZNF268f appeared to be tissue-specific. By Western blot analysis, all 3 ZNF268 protein isoforms, ZNF268a, ZNF268b1 and ZNF268b2, were expressed in tumor cell lines, while only two protein products, ZNF268b1 and ZNF268b2, were detected in human fetal tissues. Subcellular localization analysis showed that ZNF268a and ZNF268b2 distributed diffusely throughout the cell, while ZNF268b1 mainly localized in the cytoplasm. Moreover, using a CAT reporter system fused to the Gal4 DNA binding domain of the ZNF268 gene, the ZNF268a and b2 activated the CAT reporter gene expression, while the KRAB domain, corresponding to the ZNF268b1 repressed the reporter gene expression. Taken together, our results showed that multiple ZNF268 splicing products encode multiple ZNF268 protein isoforms with different subcellular localization, and that the ZNF268 gene may function as a transcriptional activator in the growth and differentiation of cells in development and/or pathogenesis.
\end{abstract}

Correspondence to: Dr Wenxin Li, State Key Laboratory of Virology, College of Life Sciences, Wuhan University, Wuhan 430072, P.R. China

E-mail: liwxlab@whu.edu.cn

Key words: alternative splicing, ZNF268, KRAB, subcellular localization, transcriptional activator

\section{Introduction}

Alternative splicing is widespread in almost all species. It is estimated that the proportion of human genes that produce alternative transcripts are as high as $74 \%$ by DNA microarray experiments $(1,2)$. The high frequency of alternative splicing in humans has also been demonstrated by expressed sequence tags (ESTs) (3-5). Studies show that alternative splicing is one of the important mechanisms regulating the expression of genes after transcription to generate cellspecific protein patterns. Unlike translational control, alternative splicing changes the structure of the mRNAs and possibly their encoded proteins, and changes in protein sequences can influence protein functions, such as binding properties, enzymatic activity, subcellular localization, and protein stability (6). The notable example of alternative splicing of multiple exons, generating up to $>30,000$ isoforms of a single gene is Dscam (axonal guidance receptor in Drosophila), which was first discovered in the nervous system mediating the formation of neuronal cell-cell contact (7), which has been found to play an important role in the immune system of insects (8).

The ZNF268 gene, cloned and characterized from a 3-5week-old human fetus of the cDNA library in our laboratory (9), is a typical KRAB-containing zinc finger gene. Evidence shows that KRAB-containing zinc finger genes usually act as a transcriptional repressor (10). Our previous studies implied that ZNF268 may play an important role in the development of human fetal liver (11). Although the exact function of the ZNF268 gene is unknown, evidence suggested there are multiple ZNF268 transcripts expressed in different organs and tissues. Recently, we cloned and characterized two novel transcripts of the ZNF268 gene in adult blood cells, which were respectively named ZNF268c and ZNF268d (12). Krackhardt and his colleagues identified $\mathrm{Kw}-4$, one of the alternative transcripts of the ZNF268 gene, as one of tumor-associated antigens in chronic lymphocytic leukemia (13). These studies suggest that by means of alternative splicing, ZNF268 may play multiple roles in the development of human fetal liver and the differentiation of blood cells.

Gene expression patterns will be important in the elucidation of its biological functions. However, little is known about the ZNF268 expression patterns in other human tissues 
Table I. Primers used in this study.

\begin{tabular}{|c|c|c|}
\hline Primer name & Primer sequence ${ }^{a}$ & Location on ZNF268b \\
\hline BU1 & 5' GTTGCGAACCCTTCTGGC 3' & 39 \\
\hline BD1 & 5' TGCACTGCAGATGCTTCCGATACAT 3' & 3604 \\
\hline BUN1 & 5' ATCTGGAGGTGGAGGCAGTA 3' & 62 \\
\hline $3 u$ & 5' TCCGGATCCGAAACAGTTCATGGG 3' & 224 \\
\hline $3 d$ & 5' TTGCTCGAGAAACAGCCACTCTAGG 3' & 387 \\
\hline $6 \mathrm{~d}$ & 5' AGAGCTCGAGGCAACAAGGCGTC 3' & 739 \\
\hline $5 \mathrm{a}$ & 5' GGACAGGTCTGATTTGGAACTTGGG 3' & 635 \\
\hline BDN1 & 5' ATGTACCTGAAACCCATTAGGAT 3' & 976 \\
\hline 268.1 & 5' GCGAGATCTACAAAACTGACCGTAGG 3' & 154 \\
\hline 268.2 & 5' TGAGTCGACGATTCCACTTACAGAG 3' & 3219 \\
\hline space 1 & 5' ATTAGATCTCTTATGGATTGGC 3' & 758 \\
\hline space2 & 5' CATGTCGACGCACATAAGTTGC 3' & 1093 \\
\hline GEX-space1 & 5' AAGGGATCCTGGGAAGTACGGCAA 3' & 792 \\
\hline GEX-space2 & 5' GCATCCAAACTCGAGTTCGCCCATA 3' & 1120 \\
\hline BXG-268au & 5' AGGCCCGGGAAACTGACCGTAG 3' & 154 \\
\hline BXG-268b2u & 5' ATTCCCGGGCTTATGGATTGGC 3' & 790 \\
\hline BXG-268d & 5' GGCATCTAGATTCCACTTACAGAG 3' & 3219 \\
\hline B-actin1 & 5' GCTCGTCGTCGACAACGGCTC 3' & \\
\hline B-actin2 & 5' CAAACATGATCTGGGTCATCTTCTC 3' & \\
\hline
\end{tabular}

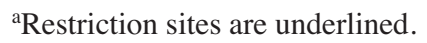

and tumor derived cells or the detailed structure and functions of different ZNF268 isoforms.

In this study, we analyzed the expression patterns and alternative splicing events of ZNF268 in human fetal tissues and several tumor derived cells by RT-PCR. Furthermore, we generated two specific anti-ZNF268 polyclonal antisera to detect the potential protein products of ZNF268. We showed that there were three protein isoforms with different subcellular localization expressed in human fetal tissues and tumor cells. In addition, transcriptional analysis suggests that ZNF268 contains transcriptional regulatory domains. Our results indicate that multiple ZNF268 transcripts can encode multiple protein isoforms with different subcellular localization, and ZNF268 may function as a transcriptional regulator in human development and pathogenesis.

\section{Materials and methods}

Human tissues. Healthy human fetus (6-month-old) was obtained from accidental abortions in the People's Hospital of Wuhan University. The study was approved by the hospital ethics committee and the family members concerned. Multiple tissues from the fetus were separated and cut into $1-\mathrm{mm}^{3}$ pieces, quickly frozen in liquid nitrogen, and stored at $-80^{\circ} \mathrm{C}$ until processed.

Cell cultures. The mammalian cell lines K562, HeLa, Jurkat, HepG2, COS7 and HEK293 were obtained from the CCTCC (Wuhan University, Hubei Province, China), and grown in a humidified $5 \% \mathrm{CO}_{2}$ atmosphere at $37^{\circ} \mathrm{C}$. K562, HeLa and Jurkat cells were cultured with RPMI-1640 medium supplemented with $10 \%$ fetal calf serum (Gibco, Invitrogen
Corporation, USA), penicillin $(50 \mathrm{U} / \mathrm{ml})$ and streptomycin (50 U/ml). HepG2, COS7, and HEK293 cells were maintained in Dulbecco's modified Eagle's medium (DMEM) supplemented with $10 \%$ fetal calf serum (Gibco, Invitrogen Corporation), penicillin $(50 \mathrm{U} / \mathrm{ml})$ and streptomycin $(50 \mathrm{U} / \mathrm{ml})$.

RNA isolation and RT-PCR. Total RNA of the cell lines or fetus tissues were prepared using TRIZOL Reagent (Gibco, Invitrogen Corporation) according to the manufacturer's instructions. To remove genomic DNA contamination, DNase treatment was performed as recommended in the manufacturer's kit. First-strand cDNA of all the samples were reverse transcribed using RNA PCR kit (Takara, Japan) with oligo dT-Adaptor primer containing $1 \mu \mathrm{g}$ of total RNA.

One-tenth of the purified DNA/RNA volume was amplified using the EX Taqase PCR system (Takara) with the primer pairs of BU1 and BD1 (Table I) for full-length ZNF268 amplification $\left(94^{\circ} \mathrm{C}\right.$ for $50 \mathrm{sec}, 58^{\circ} \mathrm{C}$ for $50 \mathrm{sec}$, and $72^{\circ} \mathrm{C}$ for $200 \mathrm{sec}$ for $\left.35 \mathrm{cycles}\right)$. The primer pairs for detection of the alternative splice events were: BUN1 and BDN1, 268.1 and 6d, 3u and BDN1, space 1 and 268.2 (Table I). PCR reactions were performed at $94^{\circ} \mathrm{C}$ for $5 \mathrm{~min}$ and then followed with $94^{\circ} \mathrm{C}, 45 \mathrm{sec} ; 58^{\circ} \mathrm{C}, 45 \mathrm{sec} ; 72^{\circ} \mathrm{C}, 90$ sec for 36 cycles followed by an extension period of $7 \mathrm{~min}$ at $72^{\circ} \mathrm{C}$. To ensure the integrity of cDNA during PCR, $\beta$-actin was amplified as a positive control with the primer pairs of $\beta$ actin 1 and $\beta$-actin2 (Table I). The PCR products were separated on a $1.5 \%$ agarose gel.

Clone and sequence of PCR products. PCR products were purified and ligated into the pGEM-T Easy Vector (Promega Corporation, USA), and then transformed into Escherichia coli. 
For each transformation of every band of PCR products, three clones were randomly picked and were determined by PCR. Representative clones identified with PCR were then sequenced.

Generation of anti-ZNF268 polyclonal antiserum. Exon3 and spacer domain of the ZNF268 gene were amplified with the primers of $3 u$ and 3d, GEX-space1 and GEX-space2, and each was inserted into prokaryotic expression vector pGEX-5X-2 (Invitrogen) to generate two prokaryotic expression plasmids. The fusion proteins were prepared from bacterial cells and purified with the GSH affinity chromatography system. The mixture of the purified proteins and Freund adjuvant (Sigma, USA) were used to generate anti-ZNF268 polyclonal antibodies in rabbits. Affinity-purified antiserum was characterized by ZNF268 protein isoforms expressed by prokaryotic expression. Rabbit pre-immune antiserum was obtained prior to immunization and used as a negative control for Western blot analysis.

Western blot analysis. Lysates of cells and total protein of tissues were prepared in lysis buffer containing $100 \mathrm{mM}$ $\mathrm{NaCl}, 10$ mM Tris-HCl (pH 8.0), 1 mM EDTA (pH 8.0), $1 \%$ Triton X-100 and $1 \mathrm{mM}$ phenyl-methylsulfonyl fluoride, followed by centrifugation at $12,000 \mathrm{~g}$ for $10 \mathrm{~min}$. Supernatants were collected, mixed with an equal volume of SDS-PAGE $2 \mathrm{X}$ sample buffer, aliquoted, and stored at $-80^{\circ} \mathrm{C}$ until used.

Aliquots $(\sim 50 \mu \mathrm{g})$ were analyzed on a $10 \%$ polyacrylamide gel and transferred to nitrocellulose membrane. The membranes were blocked with $5 \%$ non-fat dry milk for $1 \mathrm{~h}$ at $37^{\circ} \mathrm{C}$. Subsequently they were incubated with rabbit anti-ZNF268 polyclonal antiserum or pre-immune serum in a dilution $1 / 400$ for $2 \mathrm{~h}$ at $37^{\circ} \mathrm{C}$. Membranes were then incubated for $1 \mathrm{~h}$ with horseradish peroxidase conjugated goat anti-rabbit antibodies (Zhongshan Biotechnology, China) at $37^{\circ} \mathrm{C}$. The color reaction was carried out by incubation in $0.1 \mathrm{M}$ Tris- $\mathrm{HCl}(\mathrm{pH} 6.8)$, containing $0.5 \mathrm{mg} / \mathrm{ml}$ diaminobenzidine (DAB, Sigma) and $0.01 \% \mathrm{H}_{2} \mathrm{O}_{2}$. The GAPD protein was detected as a positive control.

Transient transfection and subcellular localization analysis. To study the subcellular localization of the three ZNF268 isoforms, the coding sequences of the three proteins were amplified by PCR with primer pairs (268.1 and 268.2, 268.1 and space2, space1 and 268.2, respectively) containing $B g l \mathrm{II}$ and SalI sites for cloning into the corresponding sites of the vector pEGFP (Clontech, USA) in-frame, to produce pEGFPZNF268a, pEGFP-ZNF268b1 and pEGFP-ZNF268b2, respectively. All the constructs were confirmed by sequencing. Then the constructs were transiently transfected into HEK293 and COS7 cells using Sofast ${ }^{\mathrm{TM}}$ (Sunma, China). The cells were examined after being cultured for $24-48 \mathrm{~h}$ by using a fluorescence microscope to observe the subcellular localization of the three fusion proteins. The nuclei were stained with DAPI.

Transient transfection and CAT assays. The ORF of ZNF268a and ZNF268b2 were cloned in-frame to the GAL4 DNAbinding domain (DBD) expression vector $\mathrm{pBXG1}$ at $\mathrm{SmaI} / \mathrm{XbaI}$ with primer pairs (BXG-268au and BXG-268d, BXG-268b2u and $\mathrm{BXG}-268 \mathrm{~d}$, respectively). The methods were as previously reported $(14,16)$. In brief, Plasmids pBXG1, pBXG1/ZNF268$\mathrm{KRAB}$ and $\mathrm{pBXG1/rKid1-KRAB}$ served as control. Reporter plasmid pG5SV-BCAT (200 ng) was cotransfected in COS7 cells in a 24-well plate with each of the expression vectors. Twenty-four hours later, cells were harvested and CAT activity was measured by CAT ELISA (Roche) according to the manufacturer's instructions. Each construct was analyzed in independent transfection 3 times and each time, the construct was measured in duplicate.

\section{Results}

Identification of three novel alternative splice events of ZNF268 gene in human tissues and tumor cells. Previous studies showed that the ZNF268 gene has multiple splicing products, which may play a role in the development of human liver and the differentiation of human blood cells. In order to analyze the expression pattern of the ZNF268 gene in normal human tissues and tumor cells, we designed a series of primer pairs to amplify the ZNF268 transcripts by RT-PCR from the cDNA of human fetal tissues and a series of human tumorderived cell lines. As shown in Fig. 1B, 7 distinct bands were observed when the PCR products were separated on a $1.5 \%$ agar gel. Cloning and sequencing of these products revealed 8 distinct mRNA species (Fig. 1A). Sequence alignment with ESTs in GenBank of NCBI (www.ncbi.nlm. nih.gov) indicated that they were all derived from the human ZNF268 gene. In addition to the five known transcripts of the ZNF268 gene, three novel splicing forms of the ZNF268 gene were thus identified and named ZNF268e (GenBank accession no.: DQ057358), ZNF268f (GenBank accession no.: DQ057359) and ZNF268g (GenBank accession no.: DQ057360).

Sequence analysis showed that all three novel splicing events were generated through exon skipping. As shown in Fig. 1A, ZNF268e lacks exons 4, 5 and 6; and ZNF268f is deleted of exons 3 and 4 . Both have a deletion of $328 \mathrm{nt}$ compared to the longest transcript (ZNF268b) and their RTPCR products migrated at the same location in the agar gel (Fig. 1B).

Of all 8 distinct ZNF268 transcripts, ZNF268a is the only one which encodes a full length KRAB-containing zinc finger protein with a molecular weight of $108 \mathrm{KD}$. Although ZNF268b contains all the 7 exons, there is an in-frame stop codon near the 5'-end of exon 7. It is predicted to encode ZNF268b1 protein (from the ATG in exon2 to TGA stop codon in spacer domain, near the 5'-end of exon 7). ZNF268c, ZNF268d, ZNF268e, ZNF268f, ZNF268g and $K w-4$ are predicted to be translated into a protein product of $\sim 88 \mathrm{KD}$ with spacer domain and $24 \mathrm{C} 2 \mathrm{H} 2$ zinc finger motifs (from the initiation codon near the 5'-end of exon 7 to the stop codon near the 3'-end of exon 7 (we named it ZNF268b2 protein). In addition, ZNF268b also contains the second open reading frame corresponding to ZNF268b2 and thus ZNF268b may encode two proteins if internal translation initiation occurs at the start codon near the 5'-end of exon 7.

ZNF268c and ZNF268f may be tissue-specific. ZNF268c, first isolated from human blood cells, could not be detected in human fetal liver by RT-PCR with the primer pair $3 \mathrm{u}$ and 


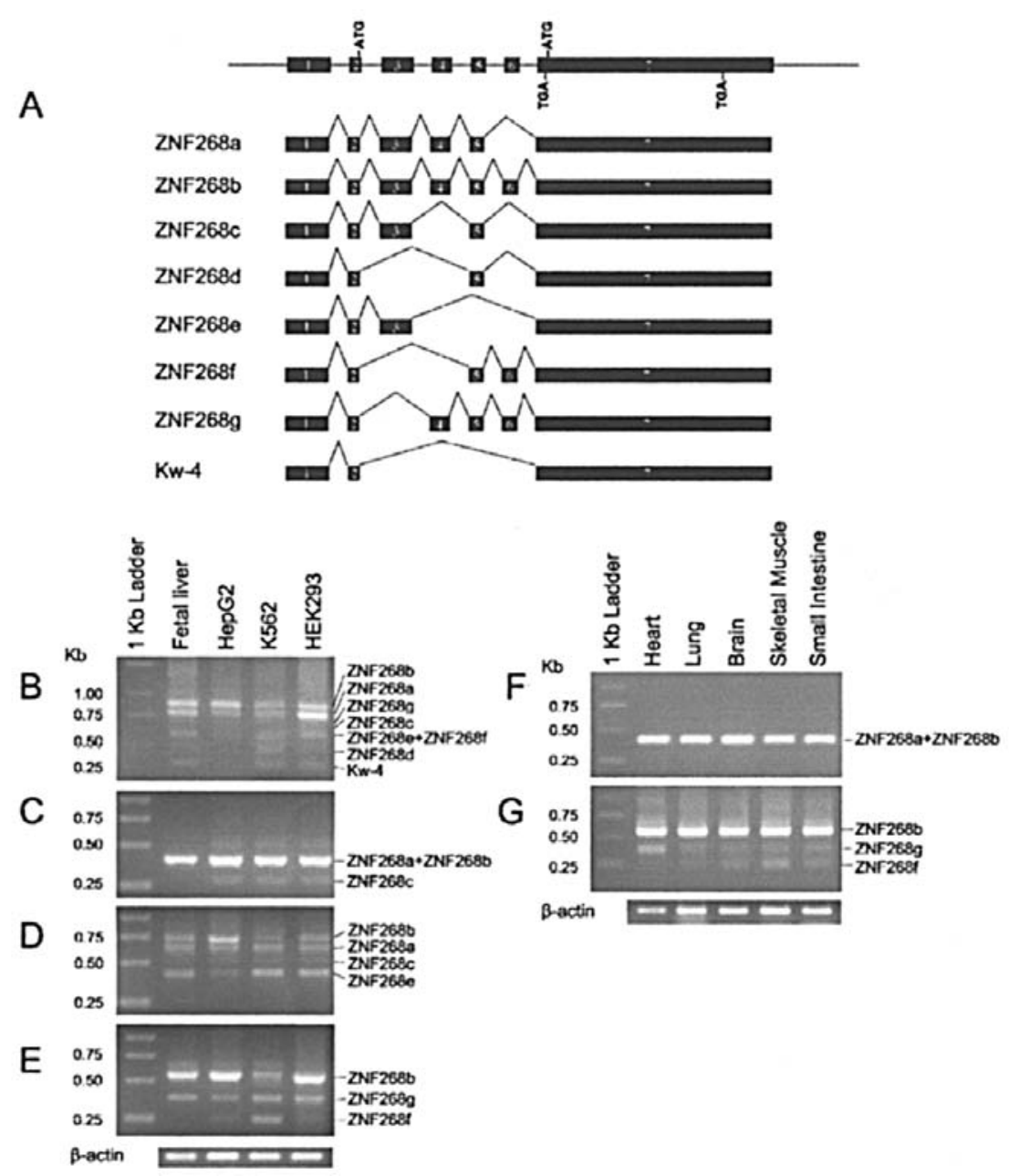

Figure 1. Alternative splicing events of ZNF268. (A) The genome structure and 8 alternatively spliced transcripts of the ZNF268 gene. The likely translation start and stop codons are indicated. (B) Detection of all different alternatively spliced transcripts by RT-PCR with the primer pair BUN1 and BUD1 in liver and 3 human tumor derived cell lines. (C and F) Analysis by RT-PCR with the primer pair 3u and 5a for ZNF268c in fetal tissues and cell lines. (D) Analysis by RT-PCR with the primer pair 3u and BDN1 for ZNF268e in tissues and cell lines. (E and G) Analysis by RT-PCR with the primer pair 268.1 and 6d for ZNF268b, ZNF268g and ZNF268f.

BDN1 (Fig. 1D) or with a different primer pair as in Fig. 1B. To further analyze the expression of ZNF268c, a specific primer pair $3 \mathrm{u}$ and $5 \mathrm{~d}$ was designed to amplify the splicing of ZNF268 in normal human tissues and tumor cells. Results showed that ZNF268c was again only detected in tumor cells, but not in normal human tissues (Fig. 1F).

RT-PCR with the oligonucleotide primer pair $3 \mathrm{u}$ and BDN1 showed that ZNF268e was expressed in human fetal liver and several tumor cell lines (Fig. 1D). RT-PCR with the primer pair 268.1 and $6 \mathrm{~d}$ revealed that ZNF268f was detected in tumor cell lines but not in human fetal liver, while ZNF268g, which lacks exon 3, was found to be expressed in all samples (Fig. 1E). Further analysis showed that ZNF268f was expressed at low levels in brain, lung and small intestine, but was not detected in fetal heart (Fig. 1G).

Expression of ZNF268 protein isoforms in human tissues and tumor cells. To determine whether the predicted protein isoforms of ZNF268 are expressed in vivo, we generated two anti-ZNF268 polyclonal antisera by using bacterium-expressed ZNF268 fragments corresponding to the E3 (exon3) and SD (spacer domain) of the ZNF268 gene. The anti-E3 polyclonal antisera should recognize ZNF268a and ZNF268b1 protein isoforms, while the anti-SD polyclonal antisera should recognize ZNF268a and ZNF268b2 protein isoforms.

We first analyzed the reactivity of anti-E3 antisera towards bacterium-expressed fusion proteins GST-E3, and GST-SD. Anti-E3 antisera were found to specifically recognize GST-E3, but not GST-SD. While polycolonal antisera of anti-SD can be reacted with GST-SD, but not with GST-E3 (Fig. 2C). Thus, both antisera are specific.

Using the anti-SD polyclonal antisera, we detected three distinct bands of $\sim 108,88$ and $60 \mathrm{KD}$ (Fig. 2D) in Jurkat, K562 and HeLa cells. The bands of 108 and $88 \mathrm{KD}$ were of the expected sizes for ZNF268a and ZNF268b2, respectively. We also detected the 88 - and $60-\mathrm{KD}$ bands but not the 108-KD band in 5 human fetus tissues. These results suggest that ZNF268a may express at a level in fetal tissues that was too low to be detected under our conditions. The $60 \mathrm{KD}$ band is likely a nonspecific band.

The exon 3 of ZNF268 is present in both ZNF268b1 and ZNF268a protein isoforms. As shown in Fig. 2E, the anti-E3 


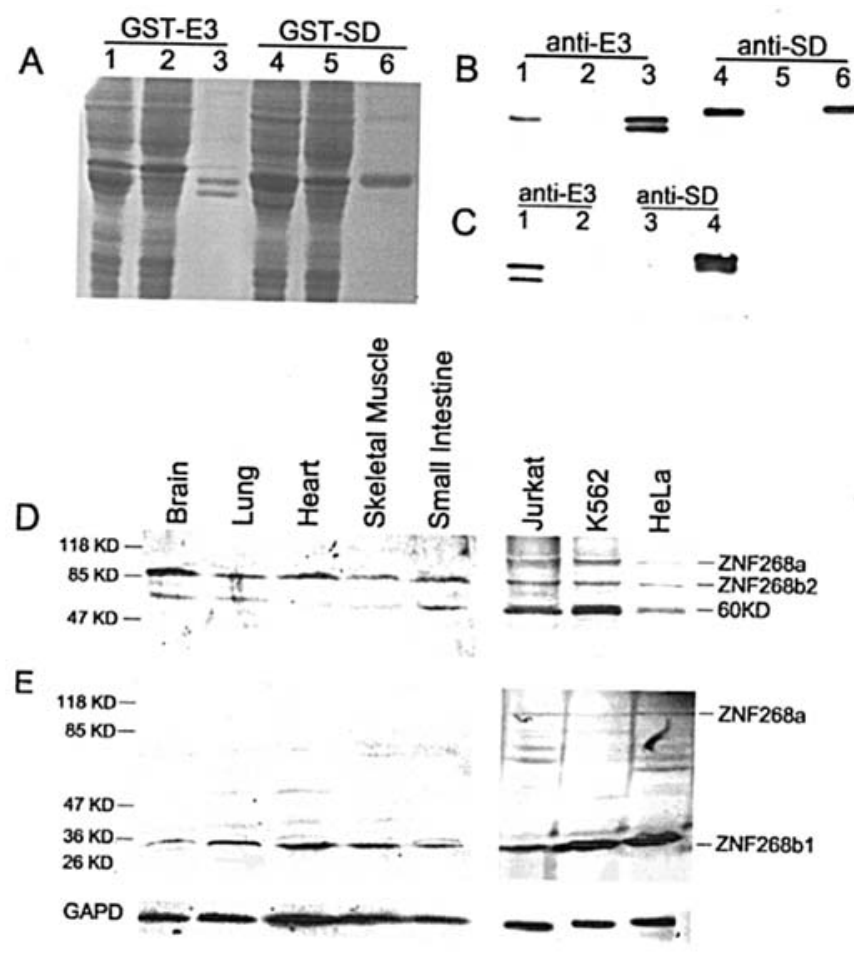

Figure 2. All three forms of ZNF268 proteins are expressed in cell lines and tissues. (A) GST-E3 and GST-SD fusion proteins were overexpressed in E. coli after IPTG induction. The whole cell lysate of the induced cell (lanes 1 and 4), uninduced cells (lanes 2 and 5) and affinity-purified ZNF268 proteins from the induced cells (lanes 3 and 6) were separated by SDSPAGE electrophoresis, and stained. (B) Proteins in gels as shown in A were transferred to nitrocellulose membrane and then blotted with anti-E3 and anti-SD polycolonal antisera as indicated, demonstrating the specificity of the respective antibodies. (C) GST-E3 (lanes 1 and 3), GST-SD (lanes 2 and 4), were separated by SDS-PAGE electrophoresis, transferred to nitrocellulose membrane, and subjected to Western blot analysis with either anti-E3 antisera or anti-SD antisera. (D) Western blot analysis of the total proteins extracted from tissues and cell lines with the polyclonal antisera of spacer domain of ZNF268 (anti-SD). Note that proteins of expected sizes for ZNF268a and $268 \mathrm{~b} 2$ were detected in all samples, consistent with the expression of the mRNA. The $60-\mathrm{kd}$ band is likely a nonspecific protein detected by the antisera. (E) Identical blot as in (D) was probed with the polyclonal antisera against exon3 (anti-E3) of ZNF268. GAPD was detected as a loading control with anti-GAPD. Pre-immune antibodies against SD and E3 failed to detect any bands (data not shown).

polyclonal antisera detected one main band at $\sim 36 \mathrm{KD}$, expected size of ZNF268b1, in the tissues and tumor cells. In addition, a band the size of ZNF268a could also be detected in tumor cell lines but not in normal tissues, in agreement with the findings of the anti-SD polyclonal antisera.

The proteins of ZNF268a, ZNF268b1 and ZNF268b2 have different subcellular localization. As subcellular localization is a critical aspect that determines the function of the proteins, we analyzed the subcellular localization of the three ZNF268 proteins. We fused all three isoforms to EGFP in an expression vector. Transient transfection studies showed that both ZNF268a- and ZNF268b2-EGFP fusion proteins distributed diffusely in COS7 cells (Fig. 3B). Although a weak fluorescence was observed in the transfected nuclei, ZNF268b1 was mainly observed in the cytoplasm (Fig. 3B). The same localization pattern was observed for each isoform regardless whether the EGFP was fused to the $\mathrm{NH} 2$ - or $\mathrm{COOH}$-terminus

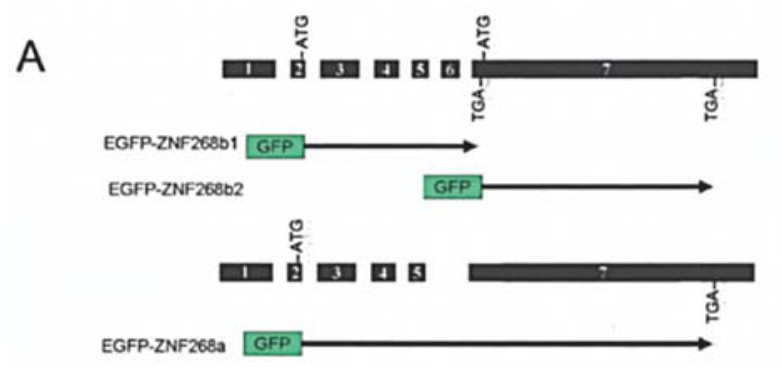

B

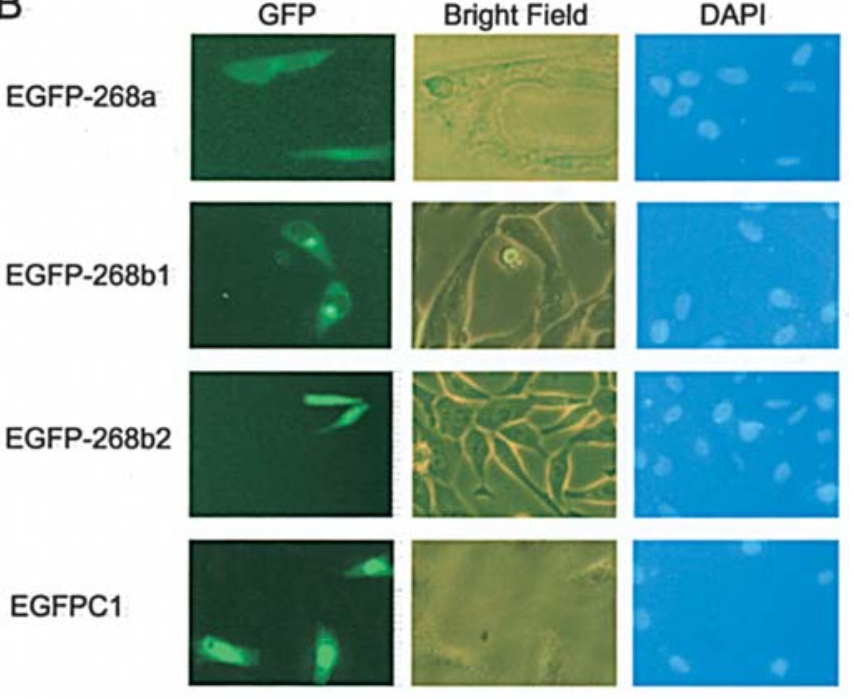

Figure 3. Subcellular localization of the three ZNF268 isoforms. (A) The three EGFP-ZNF268 fusion proteins. (B) COS7 cells were transiently transfected with the three EGFP fusion protein constructs as indicated and were examined after culturing for $24-48 \mathrm{~h}$ by using a fluorescent microscope to observe the subcellular localization of the three fusion proteins. DAPI stain was used to confirm the location of the nucleus.

(data not shown), suggesting that the subcellular localization patterns reflected the native ZNF268 isoforms. The ZNF268b1 fusion protein largely appeared as dot-like aggregates in cytoplasm, while the ZNF268b2 fusion proteins had dot-like aggregates in nuclei. Similar subcellular localization patterns of these GFP-fusion proteins were also observed in HEK293 cells (data not shown).

Transcriptional regulatory activity of ZNF268 isoforms. Previous studies have indicated that the KRAB domain of ZNF268, which is essentially equivalent to ZNF268b1, has transcriptional repressor activity (14). However, the transcriptional activity of ZNF268a and ZNF268b2 remains unknown. In addition, most of studies have shown that KRABcontaining zinc finger protein function as transcriptional repressors, but only a few are characterized as transcriptional activators (15). Thus, we used a CAT reporter system to investigate the transcriptional activity of the ZNF268 isoforms. As reported previously $(14,16)$, the CAT reporter plasmid pG5SV-BCAT contains five GAL4-binding elements and exhibits a basal level of transcriptional activity in the presence of cotransfected control expression plasmid pBXG1. In a dose-dependent manner, pBXG1/ZNF268-KRAB and pBXG1/rKid1-KRAB strongly repressed the expression of 
A

Expression plasmids

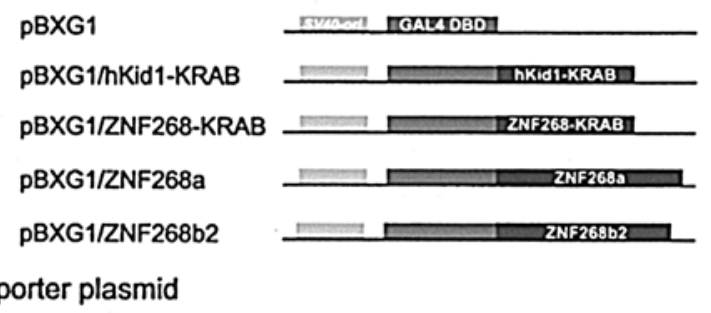

PG5SV-BCAT

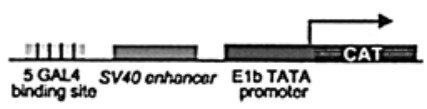

B

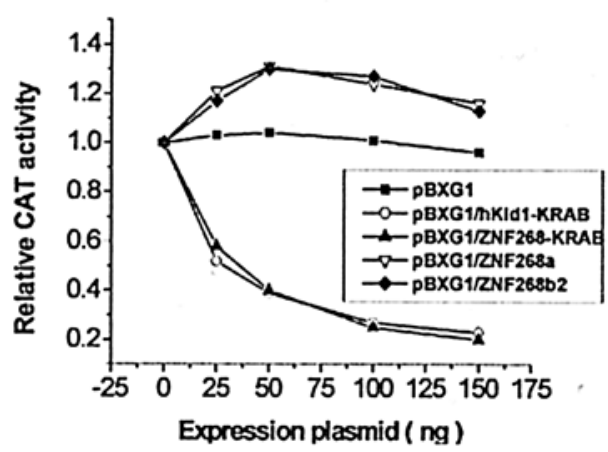

Figure 4. ZNF268 proteins contain transcription regulatory activity. (A) Expression constructs and the reporter plasmid used in the transfection assay. (B) Relative CAT activity of the reporter gene in the presence of various expression plasmids. Different amounts of the indicated expression plasmid were cotransfected with $200 \mathrm{ng}$ pG5SV-BCAT reporter plasmid in 24-well plates. Cells were harvested after $24 \mathrm{~h}$ and CAT activity was measured by CAT ELISA (Roche) according to manufacturer's instructions.

CAT (14). On the other hand, pBXG1/ ZNF268a and pBXG1/ZNF268b2 activated the expression of the reporter gene (Fig. 4). These results suggest that ZNF268a and ZNF268b2 may function as transcriptional activators while ZNF268b1 as a transcription repressor.

\section{Discussion}

Alternative splicing is an important mechanism for gene regulation and function in normal development as a means of creating protein diversity in complex organisms. It also has been found that aberration of alternative splicing without genomic mutation was one of the important causes for cancer development (17-19). In this study, we used RT-PCR to examine the expression pattern of ZNF268 mRNAs in human fetal tissues and tumor derived cell lines. In addition to the 5 transcripts of ZNF268 characterized previously, there are at least 3 additional alternative splicing products of the ZNF268 gene expressed in human tissues and tumor derived cell lines. Alternative splicing events occur mainly involving exon 3, exon 4, exon 5, and exon 6. The KRAB domain of ZNF268 is encoded by exon 4 and exon 5 . Those ZNF268 splicings with deletion of exon 4 and/or exon 5 can not be translated into a KRAB-containing zinc finger protein. Thus, alternative splicing may contribute to the diversity of the function of ZNF268.
Examination of the multiple ZNF268 transcripts by RTPCR revealed that the expression level of different transcripts was distinct in human fetal tissues and tumor cell lines. ZNF268a and ZNF268b mRNA were expressed at relatively high levels in all samples. The other transcripts of the ZNF268 gene were expressed at lower levels. In particular, ZNF268c, which was first isolated from human blood cells (12), could be detected in tumor derived cell lines, but not in the fetal substantiality tissues. This indicated that the expression of ZNF268c mRNA may be associated with tumorigenesis. $Z N F 268 f$ is another interesting transcript. Its expression also shows tissue specificity.

Alternative splicing allows for a single gene to encode a functionally or structurally diverse family of proteins (17). By Western blot, we showed that all three predicted ZNF268 protein isoforms were present in human tumor derived cell lines but only two protein products could be detected in human fetus tissues. ZNF268a protein could be detected in all tested human tumor derived cell lines but not in normal fetus tissues. ZNF268a was the only transcript that can be translated into a typical KRAB-containing zinc finger protein. The KRAB domain of ZNF268 has been shown to be a strong transcriptional repressor (14). ZNF268b2, which lacks the KRAB domain, may play a different role in the development of human embryo and the generation of tumors.

ZNF268a and ZNF268b2 have similar subcellular localization patterns. Both are distributed in the cytoplasm and the nucleus, although the nuclear level appears to be lower. In contrast, ZNF268b1 is present predominantly in the cytoplasm. Transient transfection studies suggest that both ZNF268a and ZNF268b2 have transcriptional activation properties. As transcriptional co-regulators, most of KRABcontaining zinc finger proteins display nucleus localization. They bind specifically to a gene promoter through their multiple zinc finger motifs, and recruit some corepressors to mediate transcriptional repression (20). On the other hand, similar to ZNF268, a few other KRAB-containing zinc finger proteins are known to function as transcriptional activators. Among them are ZNF322 (21), ZNF445 (22) and K-RBP (23).

As transcriptional factors, KRAB-containing zinc finger proteins are thought to play critical roles to affect cell differentiation, cell proliferation, apoptosis and neoplasitc transformation. The ZNF268 gene, which is expressed in human fetal tissues and tumor cells, has multiple transcripts and protein isoforms with different subcellular localization. While two of the three forms have transcriptional activator function, the third, ZNF268b1, has repressor activity when fused to a DNA binding domain. On the other hand, ZNF268b1 appears to be cytoplasmic. Further studies, especially at functional level in vivo, are needed to clarify the function of different isoforms of ZNF268 in development and pathogenesis.

\section{Acknowledgements}

We thank Dr Yunbo Shi (NIH) for helpful advice and critically reading of this study. We are also grateful to Dr Maurizio Crestani for plasmid information. This study was supported by the National Natural Science Foundation of 
China (No. 30500266) and the Research Fund for the Doctoral Program of Higher Education (No. 20020486044).

\section{References}

1. Johnson JM, Castle J, Garrett-Engele P, Kan Z, Loerch PM, et al: Genome-wide survey of human alternative pre-mRNA splicing with exon junction microarrays. Science 302: 2141-2144, 2003.

2. Modrek B and Lee C: A genomic view of alternative splicing. Nat Genet 30: 13-19, 2002.

3. Modrek B, Resch A, Grasso C and Lee C: Genome-wide detection of alternative splicing in expressed sequences of human genes. Nucleic Acids Res 29: 2850-2859, 2001.

4. Kan Z, Rouchka EC, Gish WR and States DJ: Gene structure prediction and alternative splicing analysis using genomically aligned ESTs. Genome Res 11: 889-900, 2001.

5. Brett D, Brett D, Hanke J, Lehmann G, Haase S, et al: EST comparison indicates $38 \%$ of human mRNAs contain possible alternative splice forms. FEBS Lett 474: 83-86, 2000.

6. Stamm S, Ben-Ari S, Rafalska I, Tang YS, et al: Function of alternative splicing. Gene 344: 1-20, 2005.

7. Schmucker D, Clemens JC, Shu H, Worby CA, Xiao J, et al: Drosophila Dscam is an axon guidance receptor exhibiting extraordinary molecular diversity. Cell 101: 671-684, 2000.

8. Watson FL, Puttmann-Holgado R, Thomas F, Lamar DL, et al: Extensive diversity of Ig-superfamily proteins in the immune system of insects. Science 309: 1874-1878, 2005.

9. Gou DM, Sun Y, Gao L, Chow LMC, Huang J, et al: Cloning and characterization of a novel Kruppel-like zinc finger gene, ZNF268, expressed in early human embryo. BBA 1518: 306-310, 2001.

10. Gebelein B and Urrutia R: Sequence-specific transcriptional repression by $\mathrm{KS} 1$, a multiple-zinc-finger-Kruppel-associated box protein. Mol Cell Biol 21: 928-939, 2001.

11. Sun Y, Shao HJ, Li Z, Liu J, Gao L, et al: ZNF268, a novel Kruppel-like zinc finger protein, is implicated in early human liver development. Int J Mol Med 14: 971-975, 2004.
12. Sun C, Zhao ZZ, Gao L, Sun Y, Shao HJ and Li WX: Cloning and characterization of two novel transcripts of ZNF268 in human blood cells. Yichuan (In press)

13. Krackhardt AM, Witzens M, Harig S, Hodi FS, et al: Identification of tumor-associated antigens in chronic lymphocytic leukemia by SEREX. Blood 100: 2123-2131, 2002.

14. Sun Y, Gou DM, Liu H, Peng X and Li WX: The KRAB domain of zinc finger gene ZNF268: a potential transcriptional repressor. IUBMB Life 55: 127-131, 2003.

15. Dang DT, Pevsner J and Yang VW: The biology of the mammalian Kruppel-like family of transcription factors. Int $\mathbf{J}$ Biochem Cell Biol 32: 1103-1121, 2001.

16. Gao L, Sun C, Qiu HL, Liu H, Shao HJ, et al: Cloning and characterization of a novel human zinc finger gene, hKid3, from a C2H2-ZNF enriched human embryonic cDNA library. Biochem Biophy Res Commun 325: 1145-1152, 2004.

17. Black DL: Mechanisms of alternative pre-messenger RNA splicing. Annu Rev Biochem 72: 291-336, 2003.

18. Garcia-Blanco MA, Baraniak AP and Lasda EL: Alternative splicing in disease and therapy. Nat Biotechnol 22: 535-546, 2004.

19. Faustino NA and Cooper TA: Pre-mRNA splicing and human disease. Genes Dev 17: 419-437, 2003.

20. Urrutia R: KRAB-containing zinc-finger repressor proteins. Genome Biol 4: 231, 2003.

21. Li YQ, Wang YQ, Zhang CB, et al: ZNF322, a novel human $\mathrm{C} 2 \mathrm{H} 2$ Krüppel-like zinc-finger protein, regulates transcriptional activation in MAPK signaling pathways. Biochem Biophy Res Commun 325: 1383-1392, 2004

22. Luo K, Yuan J, Shan Y, Li J, Xu M, et al: Activation of transcriptional activities of AP1 and SRE by a novel zinc finger protein ZNF445. Gene 367: 89-100, 2006.

23. Wang S, Liu S, Wu MH, Geng Y and Wood C: Identification of a cellular protein that interacts and synergizes with the RTA (ORF50) protein of Kaposi's sarcoma-associated herpesvirus in transcriptional activation. J Virol 75: 11961-11973, 2001. 